SFB

Optimal designs for

trigonometric regression

models

Holger Dette, Viatcheslav B. Melas,

Petr Shpilev

Nr. 14/2009

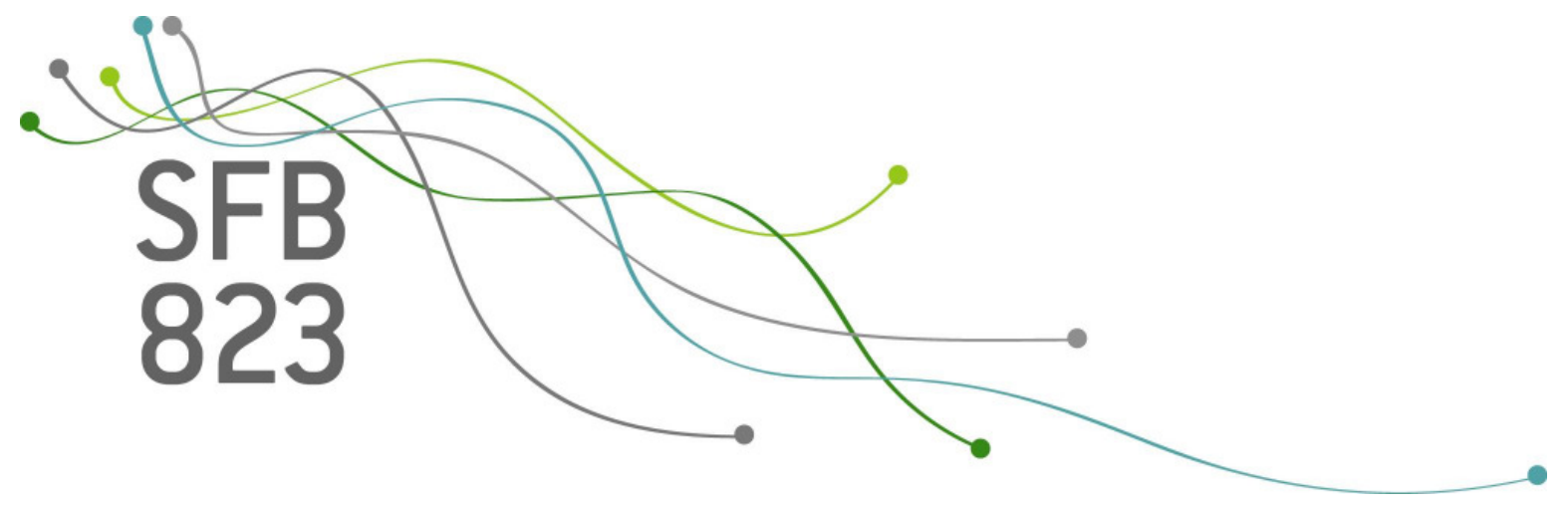





\title{
Optimal designs for trigonometric regression models
}

\author{
Holger Dette \\ Ruhr-Universität Bochum \\ Fakultät für Mathematik \\ 44780 Bochum, Germany \\ e-mail: holger.dette@rub.de
}

\author{
Viatcheslav B. Melas \\ St. Petersburg State University \\ Department of Mathematics \\ St. Petersburg, Russia \\ email: v.melas@pobox.spbu.ru
}

Petr Shpilev

St. Petersburg State University

Department of Mathematics

St. Petersburg, Russia

email: pitshp@hotmail.com

4 July 2009

\begin{abstract}
In the common Fourier regression model we investigate the optimal design problem for the estimation of linear combinations of the coefficients, where the explanatory variable varies in the interval $[-\pi, \pi]$. In a recent paper Dette et. al. (2008) determined optimal designs for estimating certain pairs of the coefficients in the model. The optimal design problem corresponds to a linear optimality criterion for a specific matrix $L$. In the present paper these results are extended to more general matrices $L$. By our results the optimal design problem for a Fourier regression of large degree can be reduced to a design problem in a model of lower degree, which allows the determination of $L$-optimal designs in many important cases. The results are illustrated by several examples.
\end{abstract}

Keywords and Phrases: L-optimal designs, Fourier regression models, parameter subsets, equivalence theorem

AMS Subject classification: $62 \mathrm{~K} 05$ 


\section{Introduction}

Consider the common Fourier or trigonometric regression model

$$
y=\beta^{T} f(t)=\beta_{0}+\sum_{j=1}^{m} \beta_{2 j-1} \sin (j t)+\sum_{j=1}^{m} \beta_{2 j} \cos (j t)+\varepsilon,
$$

where $\beta=\left(\beta_{0}, \ldots, \beta_{2 m}\right)^{T}$ denotes the vector of unknown parameters,

$$
f(t)=\left(f_{0}(t), \ldots, f_{2 m}(t)\right)^{T}=(1, \sin t, \cos t, \ldots, \sin (m t), \cos (m t))^{T} .
$$

is the vector of regression functions. The explanatory variable $t$ varies in the compact interval $[-\pi, \pi]$ and observations under different experimental conditions are assumed to be independent. An approximate design is defined as a probability measure $\xi$ on the design space $[-\pi, \pi]$ with finite support [see Kiefer (1974)]. The support points of the design $\xi$ give the locations, where observations are taken, while the weights give the corresponding proportions of the total number of observations to be taken at these points. If the designs $\xi$ puts masses $\xi_{i}$ at the points $t_{i}(i=1, \ldots, k)$ and $N$ uncorrelated observations can be taken, then the quantities $\xi_{i} N$ are rounded to integers such that $\sum_{i=1}^{k} n_{i}=N$ [for an rounding procedure - see e.g. Pukelsheim and Rieder (1992)] and the experimenter takes $n_{i}$ observations at each $t_{i}(i=1, \ldots, k)$. In this case the covariance matrix of the least squares estimator for the parameter $\beta$ in the trigonometric regression model (1.1) is approximately given by $\frac{\sigma^{2}}{N} M^{-1}(\xi)$, where

$$
M(\xi)=\left(\int_{-\pi}^{\pi} f(t) f^{T}(t) d \xi(t)\right) \in \mathbb{R}^{2 m+1 \times 2 m+1}
$$

denotes the information matrix of the design $\xi$. An optimal design maximizes a statistical meaningful (concave) function of the information and numerous criteria have been proposed in the literature [see Silvey (1980), Pázman (1986), Pukelsheim (1993)]. Because the choice of an appropriate design can improve the efficiency of the statistical analysis substantially optimal design problems for trigonometric regression model have been discussed by numerous authors [see e.g. Karlin and Studden (1966), page 347, Hill (1978) or Wu (2002), Pukelsheim (1993), p. 241, Lau and Studden (1985), Biedermann, Dette Hoffmann (2007), Dette and Haller (1998) and Zen and Tsai (2004) among others]. More recent work discussed the problem of construction optimal designs for the estimation of particular coefficients in the Fourier regression model (1.1) [see Dette and Melas (2003), Dette et. al. (2007) and Dette et. al. (2008)].

In the last named reference optimal designs have been determined, which minimize $\operatorname{tr}\left(L M^{-1}(\xi)\right)$, where the $(2 m+1) \times(2 m+1)$ matrix $L$ is given by

$$
\begin{aligned}
L_{\left(2\left\lfloor\frac{m}{2}\right\rfloor-1,4\left\lfloor\frac{m}{2}\right\rfloor-1\right)} & =e_{2\left\lfloor\frac{m}{2}\right\rfloor-1} e_{2\left\lfloor\frac{m}{2}\right\rfloor-1}^{T}+e_{4\left\lfloor\frac{m}{2}\right\rfloor-1} e_{4\left\lfloor\frac{m}{2}\right\rfloor-1}^{T} \\
L_{\left(2\left\lfloor\frac{m}{2}\right\rfloor, 4\left\lfloor\frac{m}{2}\right\rfloor\right)} & =e_{2\left\lfloor\frac{m}{2}\right\rfloor} e_{2\left\lfloor\frac{m}{2}\right\rfloor}^{T}+e_{4\left\lfloor\frac{m}{2}\right\rfloor} e_{4\left\lfloor\frac{m}{2}\right\rfloor}^{T}
\end{aligned}
$$


and $e_{j}$ denote the $(j+1)$ th unit vector in $\mathbb{R}^{2 m+1}$. $L$-optimal designs for the matrices defined in (1.3) and (1.4) allow a precise estimation of a specific pair of coefficients $\left\{\beta_{2\left\lfloor\frac{m}{2}\right\rfloor-1}, \beta_{4\left\lfloor\frac{m}{2}\right\rfloor-1}\right\}$ and $\left\{\beta_{2\left\lfloor\frac{m}{2}\right\rfloor}, \beta_{4\left\lfloor\frac{m}{2}\right\rfloor}\right\}$, respectively. Dette et. al. (2008) also determined $L$-optimal for estimating one of the pairs of the coefficients $\left\{\beta_{2 k-1}, \beta_{4 k-1}\right\},\left\{\beta_{2 k-1}, \beta_{6 k-1}\right\},\left\{\beta_{4 k-1}, \beta_{6 k-1}\right\}$, $\left\{\beta_{0}, \beta_{2 k}\right\},\left\{\beta_{0}, \beta_{4 k}\right\},\left\{\beta_{0}, \beta_{6 k}\right\},\left\{\beta_{2 k}, \beta_{4 k}\right\},\left\{\beta_{2 k}, \beta_{6 k}\right\}$ or $\left\{\beta_{4 k}, \beta_{6 k}\right\}$ in a Fourier regression model (1.1) of degree $m=3 k$ and one pair of the coefficients $\left\{\beta_{2 k-1}, \beta_{4 k-1}\right\},\left\{\beta_{2 k-1}, \beta_{6 k-1}\right\}$, $\left\{\beta_{2 k-1}, \beta_{8 k-1}\right\},\left\{\beta_{4 k-1}, \beta_{6 k-1}\right\},\left\{\beta_{4 k-1}, \beta_{8 k-1}\right\},\left\{\beta_{6 k-1}, \beta_{8 k-1}\right\},\left\{\beta_{0}, \beta_{2 k}\right\},\left\{\beta_{0}, \beta_{4 k}\right\},\left\{\beta_{0}, \beta_{6 k}\right\}$, $\left\{\beta_{0}, \beta_{8 k}\right\},\left\{\beta_{2 k}, \beta_{4 k}\right\},\left\{\beta_{2 k}, \beta_{6 k}\right\},\left\{\beta_{2 k}, \beta_{8 k}\right\},\left\{\beta_{4 k}, \beta_{6 k}\right\},\left\{\beta_{4 k}, \beta_{8 k}\right\},\left\{\beta_{6 k}, \beta_{8 k}\right\}$ in the model of degree $m=4 k$. These authors also demonstrated that the uniform designs are optimal for estimating a subset of the coefficients $\left\{\beta_{2 i_{1}-1}, \beta_{2 i_{1}}, \ldots, \beta_{2 i_{r}-1}, \beta_{2 i_{r}}\right\}$, where $1 \leq i_{1}<\ldots<$ $i_{r} \leq m, r \in\{1, \ldots, m\}$.

The present paper is devoted to some generalizations of these results. More precisely, we investigate $L$-optimal design problems for estimating more general classes of linear combinations of the coefficients. In Section 2, we introduce the basic notation and state several preliminary results, which are useful for the proof of our main results in Section 3. In particular, we characterize the structure of the pseudo-inverse of the information matrix of symmetric $L$-optimal designs. These results allow us to reduce the optimal design problem in a trigonometric regression model of large degree to an optimal design problem in a trigonometric model of relatively small degree. This observation is particularly useful for the determination of optimal designs, because the reduced optimal design problem can usually be solved analytically. Finally, several examples and extensions are presented in Section 4 in order to illustrate the theoretical results.

\section{$2 \quad L$-optimal designs}

In this Section we state several results, which turn out to be useful for the calculation of $L$-optimal designs in the trigonometric regression model (1.1). Most of the results can be found in Dette et. al. (2008) or are proved by similar arguments. The proofs of the following statements are therefore omitted. Recall the definition of the vector $f(t)$ of regression functions in Section 1 and note, that for a symmetric design $\xi$ after an appropriate permutation $P \in R^{2 m+1 \times 2 m+1}$ of the order of the regression functions the information matrix (2.1) will be block diagonal, that is

$$
\widetilde{M}(\xi)=P M(\xi) P=\left(\begin{array}{cc}
M_{c}(\xi) & 0 \\
0 & M_{s}(\xi)
\end{array}\right)
$$


where the blocks are given by

$$
\begin{aligned}
& M_{c}(\xi)=\left(\int_{-\pi}^{\pi} \cos (i t) \cos (j t) d \xi(t)\right)_{i, j=0}^{m} \\
& M_{s}(\xi)=\left(\int_{-\pi}^{\pi} \sin (i t) \sin (j t) d \xi(t)\right)_{i, j=1}^{m}
\end{aligned}
$$

For a given matrix

$$
L=\sum_{i=0}^{2 m} l_{i} l_{i}^{T},
$$

with vectors $l_{i} \in \mathbb{R}^{2 m+1}$ the class $\Xi_{L}$ is defined as the set of all approximate designs for which linear combinations of the parameters $l_{i}^{T} \beta, i=0, \ldots, 2 m$ are estimable, that is $l_{i} \in$ Range $(M(\xi)) ;(i=0, \ldots, 2 m)$. We say that an approximate design $\eta$ belongs to class $\Xi_{L}^{*}$ if $\eta \in \Xi_{L}$ and for any approximate design $\xi$ the limit

$$
\lim _{\alpha \rightarrow 0} f^{T}(t) M^{+}\left(\xi_{\alpha}\right) L M^{+}\left(\xi_{\alpha}\right) f(t)=f^{T}(t) M^{+}(\eta) L M^{+}(\eta) f(t)
$$

exists, where $\xi_{\alpha}=(1-\alpha) \eta+\alpha \xi, \alpha \in[0,1]$. Finally, a design $\xi^{*}$ is called $L$-optimal if

$$
\xi^{*}=\arg \min _{\xi \in \Xi_{L}} \operatorname{tr} L M^{+}(\xi),
$$

where $L$ is a fixed and nonnegative definite matrix and for a given matrix $A$ the matrix $A^{+}$is the Moore-Penrose inverse of $A$ [see Rao (1968)]. The following result gives a characterization of $L$-optimal designs, which is particularly useful for determining $L$-optimal designs with a singular information matrix. The theorem is stated for a general regression model $Y=$ $\beta^{T} f(t)+\varepsilon$ with $2 m+1$ regression functions. The proof can be obtained from standard arguments of approximate optimal design theory and is therefore omitted.

Theorem 2.1 Let $L \in R^{(2 m+1) \times(2 m+1)}$ denote a given and nonnegative definite matrix of the form (2.4) and assume that there exists an optimal design $\xi^{*} \in \Xi_{L}^{*}$.

1) A design $\xi$ is an element of the class $\Xi_{L}$ if and only if

$$
l_{i}^{T} M^{-}(\xi) M(\xi)=l_{i}^{T}, i=0, \ldots, 2 m .
$$

2) A design $\xi \in \Xi_{L}^{*}$ is L-optimal if and only if

$$
\max _{t \in \chi} \varphi\left(t, \xi^{*}\right)=\operatorname{tr} L M^{+}\left(\xi^{*}\right),
$$

where $\varphi(t, \xi)=f^{T}(t) M^{+}(\xi) L M^{+}(\xi) f(t)$. Moreover, the equality

$$
\varphi\left(t_{i}, \xi^{*}\right)=\operatorname{tr} L M^{+}\left(\xi^{*}\right)
$$

holds for any $t_{i} \in \operatorname{supp}\left(\xi^{*}\right)$. 
3) Assume that a design $\xi \in \Xi_{L}$ but $\xi \notin \Xi_{L}^{*}$ and these exist an interval $\left[x_{0}, b\right)$ and a family of designs $\{\xi(x)\}$ such that

$$
\begin{gathered}
\xi(x) \in \Xi_{L}^{*} \text { for } x \in\left(x_{0}, b\right), \\
\lim _{x \rightarrow x_{0}} \xi(x)=\xi
\end{gathered}
$$

and

$$
\lim _{x \rightarrow x_{0}} \max _{t \in \chi} \varphi(t, \xi(x))=\operatorname{tr} L M^{+}(\xi) .
$$

Then the design $\xi$ is L-optimal.

In general an analytical determination of $L$-optimal designs is very difficult. However, in the following Section we demonstrate that Theorem 2.1 can be used to check the optimality of a given design. The present section will be concluded with an example, which gives a more careful look at the assumption of Theorem 2.1. More precisely, we show that there exist $L$-optimal designs which do not belong to the class $\Xi_{L}^{*}$ and do not satisfy the second condition of the Theorem 2.1.

Example 2.2. We investigate the problem of constructing the $L$-optimal design for estimating the pairs of the coefficients $\beta_{2}$ and $\beta_{3}$ in the trigonometric regression model (1.1) with $m=3$. In this case the matrix $L$ in (2.4) is given by $L=e_{3}^{T} e_{3}+e_{4}^{T} e_{4}$ (all other vectors in (2.4) vanish. Consider a design $\xi_{\alpha}$ of the form

$$
\xi_{\alpha}=\xi_{\alpha}(x)=\left(\begin{array}{ccccccc}
-\pi & -\pi+x & -x & 0 & x & \pi-x & \pi \\
\frac{\alpha}{4} & \frac{1-\alpha}{4} & \frac{1-\alpha}{4} & \frac{\alpha}{2} & \frac{1-\alpha}{4} & \frac{1-\alpha}{4} & \frac{\alpha}{4}
\end{array}\right),
$$

where $\alpha \in[0,1]$. A straightforward calculation shows that the information matrix of this design in the trigonometric regression model (1.1) has the form

$$
M\left(\xi_{\alpha}\right)=\left(\begin{array}{ccccccc}
1 & 0 & 0 & 0 & m_{0,4} & 0 & 0 \\
0 & m_{1,1} & 0 & 0 & 0 & m_{1,5} & 0 \\
0 & 0 & m_{2,2} & 0 & 0 & 0 & m_{2,6} \\
0 & 0 & 0 & m_{3,3} & 0 & 0 & 0 \\
m_{0,4} & 0 & 0 & 0 & m_{4,4} & 0 & 0 \\
0 & m_{1,5} & 0 & 0 & 0 & m_{5,5} & 0 \\
0 & 0 & m_{2,6} & 0 & 0 & 0 & m_{6,6}
\end{array}\right)
$$

where the non vanishing elements in this matrix are given by $m_{i, j}=2 \sum_{k=0}^{3} f_{i}\left(t_{k}\right) f_{j}\left(t_{k}\right) \omega_{k}$ and $t_{j}, \omega_{j}$ denote the support points and corresponding weights of the design $\xi_{\alpha}$. Note that the optimal design problem for estimating the coefficients $\beta_{2}$ and $\beta_{3}$ corresponds to the minimization of the sum of the elements in the position $(2,2)$ and $(3,3)$ of the Moore-Penrose 
inverse $M^{+}\left(\xi_{\alpha}\right)$. In the following we denote these elements by $d_{2,2}$ and $d_{3,3}$, respectively. It is easy to see that for a symmetric design $\xi_{\alpha}$ after an appropriate permutation $P \in R^{7 \times 7}$ the information matrix $M\left(\xi_{\alpha}\right)$ will be block diagonal

$$
P M\left(\xi_{\alpha}\right) P=\left(\begin{array}{ccccccc}
m_{2,2} & 0 & m_{2,6} & 0 & 0 & 0 & 0 \\
0 & m_{3,3} & 0 & 0 & 0 & 0 & 0 \\
m_{2,6} & 0 & m_{6,6} & 0 & 0 & 0 & 0 \\
0 & 0 & 0 & 1 & 0 & m_{0,4} & 0 \\
0 & 0 & 0 & 0 & m_{1,1} & 0 & m_{1,5} \\
0 & 0 & 0 & m_{0,4} & 0 & m_{4,4} & 0 \\
0 & 0 & 0 & 0 & m_{1,5} & 0 & m_{5,5}
\end{array}\right)=\left(\begin{array}{ccc}
\bar{M}\left(\xi_{\alpha}\right) & 0 \\
0 & \bar{M}_{1}\left(\xi_{\alpha}\right)
\end{array}\right)
$$

The elements $d_{2,2}$ and $d_{3,3}$ depend only on 5 non vanishing elements of the matrix $\bar{M}\left(\xi_{\alpha}\right)$. So we can consider this matrix instead of matrix $M\left(\xi_{\alpha}\right)$, which means that we have to find a design minimizing the function

$$
\operatorname{tr} \bar{L} \bar{M}^{+}\left(\xi_{\alpha}\right), \text { where } \bar{L}=\left(\begin{array}{lll}
1 & 0 & 0 \\
0 & 1 & 0 \\
0 & 0 & 0
\end{array}\right)
$$

In our case the matrix $\bar{M}\left(\xi_{\alpha}\right)$ is given by

$$
\bar{M}\left(\xi_{\alpha}\right)=\left(\begin{array}{ccc}
1-4 \alpha+4 \cos (x)^{2} \alpha & 0 & 1-4 \alpha+4 \cos (x) \cos (3 x) \alpha \\
0 & 4 \sin (2 x)^{2} \alpha & 0 \\
1-4 \alpha+4 \cos (x) \cos (3 x) \alpha & 0 & 1-4 \alpha+4 \cos (3 x)^{2} \alpha
\end{array}\right)
$$

For this matrix the Moore-Penrose inverse $M^{+}\left(\xi_{\alpha}\right)$ has the form

$$
\bar{M}^{+}\left(\xi_{\alpha}\right)=\bar{M}^{-1}\left(\xi_{\alpha}\right)=\left(\begin{array}{ccc}
d_{1,1} & 0 & d_{1,3} \\
0 & d_{2,2} & 0 \\
d_{1,3} & 0 & d_{3,3}
\end{array}\right)
$$

where the elements of this matrix are given by

$$
\begin{aligned}
d_{1,1} & =\frac{1-2 \alpha+2 \alpha \cos (6 x)}{-2 \alpha(\cos (6 x)-\cos (2 x)-2 \cos (4 x)+2)(4 \alpha-1)}, \\
d_{1,3} & =\frac{1-4 \alpha+2 \alpha \cos (4 x)+2 \alpha \cos (2 x)}{2 \alpha(\cos (6 x)-\cos (2 x)-2 \cos (4 x)+2)(4 \alpha-1)}, \\
d_{2,2} & =\frac{1}{4\left(\alpha \sin (2 x)^{2}\right)}, \\
d_{3,3} & =\frac{1-2 \alpha+2 \alpha \cos (2 x)}{-2 \alpha(\cos (6 x)-\cos (2 x)-2 \cos (4 x)+2)(4 \alpha-1)} .
\end{aligned}
$$


The necessary condition for an extremum permits to find $\alpha^{*}$ minimizing the function $\operatorname{tr} \bar{L} \bar{M}^{+}\left(\xi_{\alpha}\right)$ as a root of the equation

$$
\frac{\partial \operatorname{tr} \bar{L} \bar{M}^{+}\left(\xi_{\alpha}\right)}{\partial \alpha}=0
$$

This yields

$$
\alpha^{*}=\alpha^{*}(x)=1-\frac{2+4 \cos (4 x)-14 \cos (2 x)}{3-11 \cos (2 x)+7 \cos (4 x)+\cos (6 x)},
$$

and the elements of the corresponding matrix $M^{+}\left(\xi_{\alpha^{*}}\right)$ are given by

$$
\begin{aligned}
d_{1,1} & =\frac{-2 \cos (8 x)+40+32 \cos (4 x)-13 \cos (6 x)+8 \cos (2 x)}{14 \cos (4 x)+33 \cos (2 x)-\cos (6 x)-2 \cos (8 x)+20}, \\
d_{1,3} & =\frac{5 \cos (2 x)+2 \cos (6 x)+17 \cos (4 x)+15}{14 \cos (4 x)+33 \cos (2 x)-\cos (6 x)-2 \cos (8 x)+20}, \\
d_{2,2} & =\frac{2 \cos (4 x)+18 \cos (2 x)+6}{10 \cos (2 x)-2 \cos (6 x)+3 \cos (4 x)+5}, \\
d_{3,3} & =\frac{-55 \cos (2 x)-4 \cos (6 x)-39 \cos (4 x)-45}{21 \cos (2 x)+13 \cos (6 x)-2 \cos (10 x)+\cos (8 x)+13+18 \cos (4 x)} .
\end{aligned}
$$

Denote $\xi(x)=\xi_{\alpha^{*}(x)}$.

The term $\operatorname{tr} \bar{L} \bar{M}^{+}(\xi(x))=\operatorname{tr} \bar{L} \bar{M}^{+}\left(\xi_{\alpha^{*}(x)}\right)$ reduces to

$$
\operatorname{tr} \bar{L} \bar{M}^{+}(\xi(x))=\frac{-2 \cos (8 x)+70+58 \cos (2 x)-11 \cos (6 x)+54 \cos (4 x)}{14 \cos (4 x)+33 \cos (2 x)-\cos (6 x)-2 \cos (8 x)+20} .
$$

It follows from the condition $0 \leq \omega_{i} \leq 1$ that $0 \leq \alpha^{*} \leq 1$, which is satisfied if $x \in$ $\left[\frac{\pi}{6}, \frac{\pi}{2}-\frac{1}{2} \arccos \left(\frac{\sqrt{65}-7}{8}\right)\right]$. It is easy to see that function $\operatorname{tr} \bar{L} \bar{M}^{+}(\xi(x))$ is increasing on this interval. Thus the minimum of this function is achieved at the point $x=\frac{\pi}{6}$.

Now consider the function

$$
\varphi(t, \xi(x))=\bar{f}^{T}(t) \bar{M}^{+}(\xi(x)) \bar{L}^{+}(\xi(x)) \bar{f}(t)
$$

where $\bar{f}^{T}(t)=(\cos (t), \sin (2 t), \cos (3 t))$. We determine the limit of this function at the point $x=\frac{\pi}{6}$, which is given by

$$
\bar{\varphi}(t)=\lim _{x \rightarrow \frac{\pi}{6}} \varphi(t, \xi(x))=\frac{16}{9} \cos ^{2}(t)+\frac{16}{27} \cos (t) \cos (3 t)+\frac{16}{9} \sin ^{2}(2 t)+\frac{4}{81} \cos ^{2}(3 t) .
$$

On the other hand, we have

$$
\lim _{x \rightarrow \frac{\pi}{6}} \operatorname{tr} \bar{L} \bar{M}^{+}(\xi(x))=\frac{8}{3} .
$$

It follows from the equation $\bar{\varphi}^{\prime}(t)=0$ that

$$
\max _{t \in[-\pi, \pi]} \bar{\varphi}(t)=\bar{\varphi}\left( \pm \frac{\pi}{6}\right)=\bar{\varphi}\left( \pm \frac{5 \pi}{6}\right)=\frac{8}{3} .
$$


Now consider a design

$$
\bar{\xi}=\lim _{x \rightarrow \frac{\pi}{6}} \xi(x)=\lim _{\alpha \rightarrow 0} \lim _{x \rightarrow \frac{\pi}{6}} \xi_{\alpha}(x)=\left(\begin{array}{cccc}
-5 \pi / 6 & -\pi / 6 & \pi / 6 & 5 \pi / 6 \\
\frac{1}{4} & \frac{1}{4} & \frac{1}{4} & \frac{1}{4}
\end{array}\right)
$$

Due to Theorem 2.1, part 3) this design is L-optimal.

The corresponding information matrix and its Moore-Penrose inverse can be calculated as

$$
\bar{M}(\bar{\xi})=\left(\begin{array}{ccc}
\frac{3}{4} & 0 & 0 \\
0 & \frac{3}{4} & 0 \\
0 & 0 & 0
\end{array}\right) \quad \text { and } \bar{M}^{+}(\bar{\xi})=\left(\begin{array}{ccc}
\frac{4}{3} & 0 & 0 \\
0 & \frac{4}{3} & 0 \\
0 & 0 & 0
\end{array}\right)
$$

Note that for the design $\bar{\xi}$ we have

$$
\varphi(t, \bar{\xi})=f^{T}(t) M^{+}(\bar{\xi}) L M^{+}(\bar{\xi}) f(t)=\frac{16}{9} \cos ^{2}(t)+\frac{16}{9} \sin ^{2}(2 t) .
$$

¿From the equation

$$
\varphi^{\prime}(t, \bar{\xi})=0 \Longleftrightarrow \frac{16}{9}(2 \sin (4 t)-\sin (2 t))=0 \Longleftrightarrow \frac{64}{9} \sin (2 t)\left(\cos (2 t)-\frac{1}{4}\right)=0
$$

it follows that

$$
\left.\max _{t \in[-\pi, \pi]} \varphi(t, \bar{\xi})=\varphi\left(\frac{1}{2} \arccos \left(\frac{1}{4}\right)\right), \bar{\xi}\right)=\frac{25}{9}>\operatorname{tr} L M^{+}(\bar{\xi})=\frac{8}{3},
$$

and condition (2.5) in the equivalence Theorem 2.1 is not satisfied.

\section{Some solutions of the $L$-optimal design problem}

In this section we state a result which characterizes the structure of the Moore-Penrose inverse of the information matrix for several symmetric $L$-optimal designs. The following theorem can also be very useful for constructing optimal designs for estimating any linear combination of the parameters in the model. More precisely, we consider the trigonometric regression model (1.1) of degree $m=N k$ and the $L$-optimal design problem for nonnegative definite matrices $L^{(k)} \in R^{(2 N k+1) \times(2 N k+1)}$ of the form

$$
\tilde{L}^{(k)}=P L^{(k)} P=\left(\begin{array}{cc}
L_{\mathrm{cos}}^{(k)} & 0 \\
0 & L_{\mathrm{sin}}^{(k)}
\end{array}\right)
$$

where $P$ is the permutation defined by $(2.1)$ and the matrices $L_{\text {cos }}^{(k)} \in \mathbb{R}^{N k+1 \times N k+1}$ and $L_{\mathrm{sin}}^{(k)} \in \mathbb{R}^{N k \times N k}$ are matrices with elements $L_{\mathrm{cos}, i j}^{(k)}$ and $L_{\mathrm{sin}, i j}^{(k)}$ are given by

$$
L_{\mathrm{cos}, u v}^{(k)}=\left\{\begin{array}{cl}
L_{\mathrm{cos}, i j}^{(1)} & \text { if } u=(i-1) k+1, v=(j-1) k+1 \\
0 & \text { else }
\end{array}\right.
$$


$(u, v=1, \ldots, N k+1 ; \quad i, j=1, \ldots, N+1)$ and

$$
L_{\sin , u v}^{(k)}=\left\{\begin{array}{cl}
L_{\sin , i j}^{(1)} & \text { if } u=i k ; v=j k \\
0 & \text { else }
\end{array}\right.
$$

$(u, v=1, \ldots, N k)$, respectively. Here $L_{\sin }^{(1)}=\left(L_{\sin , u, v}^{(1)}\right)_{u, v=1}^{N}$ and $L_{\mathrm{cos}}^{(1)}=\left(L_{\mathrm{cos}, u, v}^{(1)}\right)_{u, v=1}^{N+1}$ are given $N \times N$ and $(N+1) \times(N+1)$ matrices, respectively. As an illustration consider the case $N=2$ and a matrix $L_{\text {sin }}^{(1)} \in \mathbb{R}^{2 \times 2}$ of the form

$$
L_{\sin }^{(1)}=\left(\begin{array}{lll}
L_{\sin }^{(1)} & 11 & L_{\sin 12}^{(1)} \\
L_{\sin }^{(1)} & 12 & L_{\sin }^{(1)} 22
\end{array}\right),
$$

then we obtain for $k=2$ the matrix $L_{\text {sin }}^{(2)} \in \mathbb{R}^{4 \times 4}$

$$
L_{\text {sin }}^{(2)}=\left(\begin{array}{cccc}
0 & 0 & 0 & 0 \\
0 & L_{\sin 11}^{(1)} & 0 & L_{\sin 12}^{(1)} \\
0 & 0 & 0 & 0 \\
0 & L_{\sin 21}^{(1)} & 0 & L_{\sin 22}^{(1)}
\end{array}\right)
$$

Similarly, if the matrix

$$
L_{\cos }^{(1)}=\left(\begin{array}{lll}
L_{\cos 11}^{(1)} & L_{\cos 12}^{(1)} & L_{\cos 13}^{(1)} \\
L_{\cos 12}^{(1)} & L_{\cos 22}^{(1)} & L_{\cos 23}^{(1)} \\
L_{\cos 13}^{(1)} & L_{\cos 23}^{(1)} & L_{\cos 33}^{(1)}
\end{array}\right) \in \mathbb{R}^{3 \times 3}
$$

is the given matrix $L_{\mathrm{cos}}^{(2)}$ is obtained as

$$
L_{\cos }^{(2)}=\left(\begin{array}{ccccc}
L_{\cos 11}^{(1)} & 0 & L_{\cos 12}^{(1)} & 0 & L_{\cos 13}^{(1)} \\
0 & 0 & 0 & 0 & 0 \\
L_{\cos 12}^{(1)} & 0 & L_{\cos 22}^{(1)} & 0 & L_{\cos 23}^{(1)} \\
0 & 0 & 0 & 0 & 0 \\
L_{\cos 13}^{(1)} & 0 & L_{\cos 23}^{(1)} & 0 & L_{\cos 33}^{(1)}
\end{array}\right)
$$

Theorem 3.1 Consider trigonometric regression model (1.1) with $m=N k, N, k \in \mathbb{N}$, $N \geq 2$, and let $L$ be given by (3.1) with blocks $L_{\mathrm{cos}}^{(k)}$ and $L_{\mathrm{sin}}^{(k)}$ defined by (3.2) and (3.3), respectively.

1) Define the design

$$
\xi_{m}^{s i n}=\left(\begin{array}{ccccccc}
-t_{m} & -t_{m-1} & \ldots & -t_{1} & t_{1} & \ldots & t_{m} \\
\omega_{m} & \omega_{m-1} & \ldots & \omega_{1} & \omega_{1} & \ldots & \omega_{m}
\end{array}\right)
$$


where

$$
\begin{aligned}
t_{1} & =\frac{x_{1}}{k}, \ldots, t_{\frac{N}{2}}=\frac{x_{\frac{N}{2}}}{k}, t_{\frac{N}{2}+1}=\frac{\pi-x_{\frac{N}{2}}}{k}, \ldots, t_{N}=\frac{\pi-x_{1}}{k}, \\
t_{i} & =t_{i-N}+\frac{\pi}{k}, i=N+1, \ldots, m, \\
\omega_{1} & =\frac{z_{1}}{k}, \ldots, \omega_{\frac{N}{2}-1}=\frac{z_{\frac{N}{2}-1}}{k}, \omega_{\frac{N}{2}}=\frac{z_{\frac{N}{2}}}{k}, \omega_{\frac{N}{2}+1}=\frac{z_{\frac{N}{2}}}{k}, \ldots, \omega_{N}=\frac{z_{1}}{k}, \sum_{j=1}^{\frac{N}{2}} z_{j}=\frac{1}{4}, \\
\omega_{i} & =\omega_{i-N}, i=N+1, \ldots, m
\end{aligned}
$$

if $N$ is even, and

$$
\begin{aligned}
t_{1} & =\frac{x_{1}}{k}, \ldots, t_{\frac{N-1}{2}}=\frac{x_{\frac{N-1}{2}}}{k}, t_{\frac{N+1}{2}}=\frac{\pi}{2 k}, t_{\frac{N+3}{2}}=\frac{\pi-x_{\frac{N-1}{2}}}{k}, \ldots, t_{N}=\frac{\pi-x_{1}}{k} \\
t_{i} & =t_{i-N}+\frac{\pi}{k}, i=N+1, \ldots, m \\
\omega_{1} & =\frac{z_{1}}{k}, \ldots, \omega_{\frac{N-1}{2}}=\frac{z_{\frac{N-1}{2}}}{k}, \omega_{\frac{N+1}{2}}=\frac{1}{2 k}-2 \sum_{j=1}^{\frac{N-1}{2}} \frac{z_{j}}{k}, \omega_{\frac{N+3}{2}}=\frac{z_{\frac{N-1}{2}}}{k}, \ldots, \omega_{N}=\frac{z_{1}}{k} \\
\omega_{i} & =\omega_{i-N}, i=N+1, \ldots, m
\end{aligned}
$$

if $N$ is odd. If the matrix $L$ is of the form (3.1) with $L_{\mathrm{cos}}^{(k)}=0$, then for the design $\xi_{n}^{\text {sin }}$ the quantities $\operatorname{tr} L_{\sin }^{(k)} M_{s}^{+}\left(\xi_{n}^{s i n}\right)$ and the coefficients of the function

$$
\varphi\left(t, \xi_{n}^{\sin }\right)=f_{s}^{T}(t) M_{s}^{+}\left(\xi_{n}^{\sin }\right) L_{\mathrm{sin}}^{(k)} M_{s}^{+}\left(\xi_{n}^{\sin }\right) f_{s}(t)
$$

are independent of the value $k$ for any matrix $L_{\sin }^{(k)} \in \mathbb{R}^{m \times m}$.

2) Define the design

$$
\xi_{n}^{\cos }=\left(\begin{array}{ccccccccc}
-\pi & -t_{n-1} & \ldots & -t_{1} & 0 & t_{1} & \ldots & t_{n-1} & \pi \\
\omega_{n}-\alpha & \omega_{n-1} & \ldots & \omega_{1} & \omega_{0} & \omega_{1} & \ldots & \omega_{n-1} & \alpha
\end{array}\right)
$$

where $\alpha \in\left[0, \omega_{n}\right], n=(N+1) k$ and

$$
\begin{aligned}
t_{0} & =0, t_{1}=\frac{x_{1}}{k}, \ldots, t_{\frac{N}{2}}=\frac{x_{\frac{N}{2}}}{k}, t_{\frac{N}{2}+1}=\frac{\pi-x_{\frac{N}{2}}}{k}, \ldots, t_{N}=\frac{\pi-x_{1}}{k}, \\
t_{i+1} & =t_{i-N}+\frac{\pi}{k}, i=N, \ldots, n-1, \\
\omega_{0} & =\frac{1-4 \sum_{i=1}^{\frac{N}{2}} z_{i}}{2 k}, \omega_{1}=\frac{z_{1}}{k}, \ldots, \omega_{\frac{N}{2}-1}=\frac{z_{\frac{N}{2}-1}}{k}, \omega_{\frac{N}{2}}=\frac{z_{\frac{N}{2}}}{k} \\
\omega_{\frac{N}{2}+1} & =\frac{z_{\frac{N}{2}}}{k}, \ldots, \omega_{N}=\frac{z_{1}}{k}, \omega_{i+1}=\omega_{i-N}, i=N, \ldots, n-1
\end{aligned}
$$


if $N$ is even, and

$$
\begin{aligned}
t_{0} & =0, t_{1}=\frac{x_{1}}{k}, \ldots, t_{\frac{N-1}{2}}=\frac{x_{\frac{N-1}{2}}}{k}, t_{\frac{N+1}{2}}=\frac{\pi}{2 k}, t_{\frac{N+3}{2}}=\frac{\pi-x_{\frac{N-1}{2}}}{k}, \ldots, t_{N}=\frac{\pi-x_{1}}{k} \\
t_{i+1} & =t_{i-N}+\frac{\pi}{k}, i=N, \ldots, n-1 \\
\omega_{0} & =z_{0}, \omega_{1}=z_{1}, \ldots, \omega_{\frac{N-1}{2}}=z_{\frac{N-1}{2}}, \omega_{\frac{N+1}{2}}=\frac{1}{2 k}-\sum_{j=0}^{\frac{N-1}{2}} \frac{z_{j}}{k}, \omega_{\frac{N+3}{2}}=z_{\frac{N-1}{2}}, \ldots, \omega_{N}=z_{1}, \\
\omega_{i+1} & =\omega_{i-N}, i=N, \ldots, m
\end{aligned}
$$

if $N$ is odd. If the matrix $L$ is of the form (3.1) with $L_{\sin }^{(k)}=0$, then for the design $\xi_{n}^{\text {cos }}$ the quantities $\operatorname{tr} L_{\mathrm{cos}}^{(k)} M_{c}^{+}\left(\xi_{n}^{c o s}\right)$ and the coefficients of the function

$$
\varphi\left(t, \xi_{n}^{c o s}\right)=f_{c}^{T}(t) M_{c}^{+}\left(\xi_{n}^{c o s}\right) L_{\cos }^{(k)} M_{c}^{+}\left(\xi_{n}^{\cos }\right) f_{c}(t)
$$

are independent on the value $k$ for any matrix $L_{\mathrm{cos}}^{(k)} \in \mathbb{R}^{m+1 \times m+1}$.

Proof of Theorem 3.1. We will only prove the first part of Theorem 3.1 for even $N$, the case of a odd $N$ and the second part of the Theorem are treated similarly. The idea of the proof is to show that for fixed $N$ the information matrix $M_{s}\left(\xi_{k N}^{s i n}\right)$ has a particular structure such that many of the elements of its Moore-Penrose inverse vanish. More precisely, if $m_{s[i, j]}^{+}(\xi)$ denotes the element of the matrix $M_{s}^{+}(\xi) \in R^{m \times m}$ in the $i$-th row and $j$-th column, we will show that for any $k$ the identities

$$
\begin{gathered}
m_{s[k i, k j]}\left(\xi_{k N}^{s i n}\right)=m_{s[i, j]}\left(\xi_{N}^{s i n}\right), \quad i, j=1,2, \ldots, N \\
m_{s[i k, j]}=0, i=1,2, \ldots, N, j=1,2 \ldots, N k-1 \\
j \neq k, 2 k, \ldots,(N-1) k
\end{gathered}
$$

for the elements $m_{s[i, j]}\left(\xi_{k N}^{\sin }\right)$ of the matrix $M_{s}\left(\xi_{k N}^{s i n}\right)$ are satisfied. We begin proving the identity (3.6). By the definition of information matrix $M_{s}\left(\xi_{n}\right)$ we have

$$
m_{s[i, j]}\left(\xi_{n}\right)= \begin{cases}0 & \text { if }(i+j) \text { is odd } \\ \left.2 \sum_{l=1}^{n} \sin \left(i t_{l}\right) \sin \left(j t_{l}\right)\right) \omega_{l} & \text { otherwise }\end{cases}
$$

By the well-known trigonometric formula it follows

$$
\begin{aligned}
m_{s[k i, k j]}\left(\xi_{k N}^{s i n}\right) & \left.=2 \sum_{l=1}^{k N} \sin \left(k i t_{l}\right) \sin \left(k j t_{l}\right)\right) \omega_{l}=\sum_{l=1}^{k N}\left(\cos \left(k(i-j) t_{l}-\cos \left(k(i+j) t_{l}\right)\right)\right) \omega_{l} \\
& =\sum_{l=1}^{N}\left(\cos \left((i-j)\left(k t_{l}\right)-\cos \left((i+j)\left(k t_{l}\right)\right)\right)\right)\left(k \omega_{l}\right)=m_{s[i, j]}\left(\xi_{N}^{s i n}\right) .
\end{aligned}
$$


Now we check the second identity (3.7). If $k i+j$ is even we have

$$
\begin{aligned}
& 2 \sum_{l=1}^{N k} \sin \left(k i t_{l}\right) \sin \left(j t_{l}\right) \omega_{l}=2 \sum_{p=0}^{N-1} \sum_{l=1}^{k} \sin \left(k i t_{l N-p}\right) \sin \left(j t_{l N-p}\right) \omega_{l N-p} \\
& =2 \sum_{p=0}^{\frac{N}{2}-1} \frac{z_{p+1}}{k} \sin \left(\frac{x_{p+1}}{k}\right) \sum_{l=1}^{k}(-1)^{i(l-1)}\left(\sin \left(j t_{l N-p}\right)+\sin \left(j t_{l N-p-N / 2}\right)\right) \\
& =2 \sum_{p=0}^{\frac{N}{2}-1} \frac{z_{p+1}}{k} \sin \left(\frac{x_{p+1}}{k}\right) \sum_{l=1}^{k}(-1)^{i(l-1)} \\
& \times\left(\sin \left(j\left(\frac{\pi(l-1)}{k}+\frac{z_{p}}{k}\right)\right)+\sin \left(j\left(\frac{\pi l}{k}-\frac{z_{p}}{k}\right)\right)\right) \\
& =4(-1)^{i} \sum_{p=0}^{\frac{N}{2}-1} \frac{z_{p+1}}{k} \sin \left(\frac{x_{p+1}}{k}\right) \sum_{l=1}^{k}(-1)^{(l-1)} \\
& \times \sin \left(\frac{j}{k}\left(\pi l-\frac{\pi}{2}\right)\right) \cos \left(\frac{j}{k}\left(z_{p}-\frac{\pi}{2}\right)\right) \\
& =4(-1)^{i} \sum_{p=0}^{\frac{N}{2}-1} \frac{z_{p+1}}{k} \sin \left(\frac{x_{p+1}}{k}\right) \cos \left(\frac{j}{k}\left(z_{p}-\frac{\pi}{2}\right)\right) \\
& \times \sum_{l=1}^{k}(-1)^{(l-1)} \sin \left(\frac{j}{k}\left(\pi l-\frac{\pi}{2}\right)\right) \\
& =2(-1)^{i} \sum_{p=0}^{\frac{N}{2}-1} \frac{z_{p+1}}{k} \sin \left(\frac{x_{p+1}}{k}\right) \cos \left(\frac{j}{k}\left(z_{p}-\frac{\pi}{2}\right)\right) \\
& \times(-1)^{k+j-1} \frac{\sin \left(\frac{j \pi}{2 k}\right)-\sin \left(\frac{j \pi}{2 k}\right)}{\cos \left(\frac{j \pi}{k}\right)+1} \\
& =0 \text {, }
\end{aligned}
$$

which proves the second identity and completes the proof of the Theorem.

Note that the designs in (3.4) and (3.5) are determined by the parameters $x_{1}, x_{2}, \ldots$ and $z_{1}, z_{2}, \ldots$, which usually have to be found numerically. Theorem 3.1 is a very useful instrument for finding $L$-optimal designs, because it allows to reduce the optimal design problem for the trigonometric regression model (1.1) to a design problem in a model of substantially smaller degree. As a consequence the $L$-optimal design problem simplifies sufficiently. We will now illustrate its application in a concrete example..

Example 3.2. L-optimal design for estimating the coefficients of $\sin (40 t)$ and $\sin (50 t)$ in the Fourier regression model of degree $m=50$. 
We consider the trigonometric regression model (1.1) of degree $m=N k$ and use Theorem 3.1 to determine the $L$-optimal design for estimating the pair of coefficients $\beta_{2(N-1) k-1}$ and $\beta_{2 N k-1}$, which correspond to the coefficients of the terms $\sin ((N-1) k t)$ and $\left.\sin (N k t)\right)$. Exemplarily we will consider the case $m=50, N=5, k=10$. Theorem 3.1 allows to reduce the problem of constructing $L$-optimal design for estimating the pair of coefficients $\beta_{79}$ and $\beta_{99}$ for the model degree $m=50$ to the problem of constructing $L$-optimal design for estimating the pair of coefficients $\beta_{7}$ and $\beta_{9}$ for the model degree $m=5$. Now we can find this $L$-optimal design as a solution of the system

$$
\left\{\begin{array}{l}
\frac{\partial \operatorname{tr} L_{s i n}^{(1)} M_{s}^{+}\left(\xi_{5}^{\sin }\right)}{\partial x_{i}}=0 \\
\frac{\partial \operatorname{tr} L_{s i n}^{(1)} M_{s}^{+}\left(\xi_{5}^{\sin }\right)}{\partial z_{i}}=0
\end{array}\right.
$$

where $\xi_{5}^{\text {sin }}$ is a design with a structure is defined in Theorem 3.1. Thus we have

$$
\xi_{5}^{s i n}=\left(\begin{array}{cccccccccc}
-\pi+x_{1} & -\pi+x_{2} & -\frac{\pi}{2} & -x_{2} & -x_{1} & x_{1} & x_{2} & \frac{\pi}{2} & \pi-x_{2} & \pi-x_{1} \\
z_{1} & z_{2} & \frac{1-4 z_{1}-4 z_{2}}{2} & z_{2} & z_{1} & z_{1} & z_{2} & \frac{1-4 z_{1}-4 z_{2}}{2} & z_{2} & z_{1}
\end{array}\right),
$$

where $x_{1}=0.3519978036, x_{2}=1.020599385, z_{1}=0.1112542423, z_{2}=0.09865639359$.

It follows from Theorem 3.1 that the $L$-optimal design for estimating coefficients $\beta_{2(N-1) k-1}$ and $\beta_{2 N k-1}$ (i.e. $\beta_{79}$ and $\beta_{99}$ in our case) is given by

$$
\begin{aligned}
& \xi_{N k}^{s i n}=\xi_{50}^{s i n}=\left(\begin{array}{ccccccc}
-t_{50} & -t_{49} & \ldots & -t_{1} & t_{1} & \ldots & t_{50} \\
\omega_{50} & \omega_{49} & \ldots & \omega_{1} & \omega_{1} & \ldots & \omega_{50}
\end{array}\right) \\
& t_{1}=\frac{x_{1}}{10}, t_{2}=\frac{x_{2}}{10}, t_{3}=\frac{\pi}{20}, t_{4}=\frac{\pi-x_{2}}{10}, t_{5}=\frac{\pi-x_{1}}{10}, \\
& t_{i}=t_{i-5}+\frac{\pi}{10}, i=6, \ldots, 50 \text {, } \\
& \omega_{1}=\frac{z_{1}}{10}, \omega_{2}=\frac{z_{2}}{10}, \omega_{3}=\frac{1}{20}-\frac{z_{1}}{5}-\frac{z_{2}}{5}, \omega_{4}=\frac{z_{2}}{10}, \omega_{5}=\frac{z_{1}}{10}, \\
& \omega_{i}=\omega_{i-N}, i=N+1, \ldots, m
\end{aligned}
$$

Where $x_{1}, x_{2}, z_{1}, z_{2}$ is defined above. We finally note that a straightforward calculation yields for the function $\varphi\left(t, \xi_{79,99}^{*}\right)$ in the equivalence Theorem 2.1

$$
\begin{aligned}
\varphi\left(t, \xi_{79,99}^{*}\right)= & f^{T}(t) M^{+}\left(\xi_{79,99}^{*}\right) L M^{+}\left(\xi_{79,99}^{*}\right) f(t)= \\
= & 0.2 \sin ^{2}(30 t)-1.047213603 \sin (50 t) \sin (30 t) \\
& +1.370820397 \sin ^{2}(50 t)+2.094427187 \sin ^{2}(40 t),
\end{aligned}
$$

which is depicted in Figure 1. 


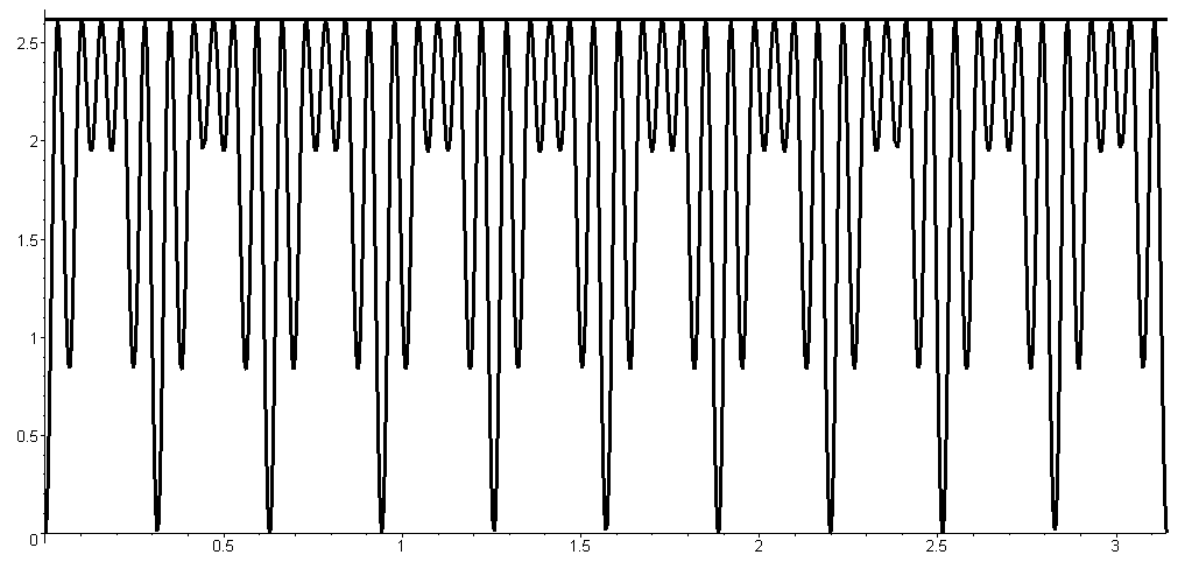

Figure 1: The function $\varphi\left(t, \xi_{79,99}^{*}\right)$ defined in the equivalence Theorem 2.1 for the L-optimal design problem discussed in Example 3.1.

\section{Further results}

In this section we present several further applications of Theorem 3.1. In particular we derive optimal designs for estimating linear combinations for estimating pairs of the coefficients in the Fourier regression model of degree $m=3 k$ (Theorem 4.1) and more general linear combinations in the model of degree $m=4 k$ (Theorem 4.2). In a recent paper Dette et. al. (2008) showed that the designs specified in (3.4) and (3.5) are optimal for estimating certain pairs of the coefficients in the trigonometric regression model (1.1) of degree $m=3 k$ and $m=4 k$. The following theorems extend these results in a non trivial manner.

Theorem 4.1 Consider trigonometric regression model (1.1) with $m=3 k$.

(1) The design $\xi_{1}^{*}=\xi_{3 k}^{\text {sin }}$ defined in (3.4) is L-optimal for estimating any pair of the coeffcients $\left\{\beta_{0}, \beta_{2 k-1}\right\},\left\{\beta_{0}, \beta_{4 k-1}\right\},\left\{\beta_{0}, \beta_{6 k-1}\right\},\left\{\beta_{2 k-1}, \beta_{4 k-1}\right\},\left\{\beta_{2 k-1}, \beta_{4 k}\right\},\left\{\beta_{2 k-1}, \beta_{6 k-1}\right\}$, $\left\{\beta_{2 k}, \beta_{6 k-1}\right\},\left\{\beta_{4 k-1}, \beta_{4 k}\right\},\left\{\beta_{4 k-1}, \beta_{6 k-1}\right\},\left\{\beta_{4 k}, \beta_{6 k-1}\right\}$.

(2) The design $\xi_{2}^{*}=\xi_{1}^{*}=\xi_{4 k}^{c o s}$ defined in (3.5) is L-optimal for estimating any pair of the coefficients $\left\{\beta_{0}, \beta_{2 k}\right\},\left\{\beta_{0}, \beta_{4 k}\right\},\left\{\beta_{0}, \beta_{6 k}\right\},\left\{\beta_{2 k-1}, \beta_{6 k}\right\},\left\{\beta_{2 k}, \beta_{4 k-1}\right\},\left\{\beta_{2 k}, \beta_{4 k}\right\}$, $\left\{\beta_{2 k}, \beta_{6 k}\right\},\left\{\beta_{4 k-1}, \beta_{6 k}\right\},\left\{\beta_{4 k}, \beta_{6 k}\right\}$.

(3) The design $\xi_{3}^{*}=\xi_{4 k}^{s i n}$ defined in (3.4) is L-optimal for estimating the pair of the coefficients $\left\{\beta_{2 k-1}, \beta_{2 k}\right\}$ and the design $\xi_{4}^{*}=\xi_{1}^{*}=\xi_{5 k}^{\text {cos }}$ defined in (3.5) is L-optimal for estimating the pair of the coefficients $\left\{\beta_{6 k-1}, \beta_{6 k}\right\}$. 
In the designs $\xi_{1}^{*}, \xi_{2}^{*}$ and $\xi_{3}^{*}$ only the values $x_{1}, x_{2}, z_{1}$ and $z_{2}$ depend on the particular pair of coefficients under consideration and are determined as the unique solution of the system

$$
\frac{\partial \operatorname{tr} L M^{+}\left(\xi^{*}\right)}{\partial x_{i}}=0 \quad \frac{\partial \operatorname{tr} L M^{+}\left(\xi^{*}\right)}{\partial z_{i}}=0
$$

The parameters determined by (3.8) are given in Table 1.

Proof of Theorem 4.1. We will only prove the first part of Theorem 4.1 for the pair $\left\{\beta_{0}, \beta_{2 k-1}\right\}$ the other cases are treated similarly. We begin with the case $k=1$, for which the design $\xi_{1}^{*}$ is given by

$$
\xi_{1}^{*}=\xi_{3}^{\sin }=\left(\begin{array}{cccccc}
-\pi+x_{1} & -\frac{\pi}{2} & -x_{1} & x_{1} & \frac{\pi}{2} & \pi-x_{1} \\
z_{1} & \frac{1-4 z_{1}}{2} & z_{1} & z_{1} & \frac{1-4 z_{1}}{2} & z_{1}
\end{array}\right)
$$

and the corresponding information matrix $M\left(\xi_{3}^{\sin }\right)$ has the form $(2.7)$. We interested in the optimal design for estimating the pair of the coefficients $\left\{\beta_{0}, \beta_{1}\right\}$, which minimizes the sum of the elements in the position $(1,1)$ and $(2,2)$ of the Moore-Penrose inverse $M^{+}\left(\xi_{3}^{\sin }\right)$. It is easy to see that these elements depend only on 6 non vanishing elements of the matrix $M\left(\xi_{3}^{\sin }\right)$. Thus we can consider the matrix

$$
\bar{M}\left(\xi_{3}^{\sin }\right)=\left(\begin{array}{cccc}
1 & 0 & m_{0,4} & 0 \\
0 & m_{1,1} & 0 & m_{1,5} \\
m_{0,4} & 0 & m_{4,4} & 0 \\
0 & m_{1,5} & 0 & m_{5,5}
\end{array}\right)
$$

instead of matrix $M\left(\xi_{3}^{s i n}\right)$. By a direct calculation we find that the optimal design for estimating the coefficients $\beta_{0}$ and $\beta_{1}$, is given by (3.9), where $x_{1}=0.6379$ and $z_{1}=0.1520$. The Moore-Penrose inverse of the corresponding information matrix can be calculated as

$$
\bar{M}^{+}\left(\xi_{1}^{*}\right)=\left(\begin{array}{cccc}
1.1169 & 0 & 0.5424 & 0 \\
0 & 1.6532 & 0 & 0.0911 \\
0.5425 & 0 & 2.5178 & 0 \\
0 & 0.0911 & 0 & 1.0788
\end{array}\right)
$$

and coincides with inverse matrix $\bar{M}^{-1}\left(\xi_{1}^{*}\right)$. Consequently the design $\xi_{1}^{*}$ belongs to class $\Xi_{L}^{*}$. Thus we can use Theorem 2.1 for checking the optimality of this design. A straightforward calculation yields for the function $\varphi_{1}\left(t, \xi_{1}^{*}\right)$

$$
\begin{gathered}
\varphi_{1}\left(t, \xi_{1}^{*}\right)=f^{T}(t) M^{+}\left(\xi_{1}^{*}\right) L M^{+}\left(\xi_{1}^{*}\right) f(t)= \\
2.7652-0.0042 \cos (2 t)-0.0035 \cos (4 t)-0.0042 \cos (6 t) \leq \operatorname{tr} L^{(1)} \bar{M}^{+}\left(\xi_{3}^{s i n}\right),
\end{gathered}
$$




\begin{tabular}{|c|c|c|c|c|c|}
\hline$m=3 k$ & $\{0,2 k-1\}$ & $\{0,2 k\}$ & $\{0,4 k-1\}$ & \multicolumn{2}{|c|}{$0,4 k$} \\
\hline$x_{1}$ & 0.6379 & 0.93293 & $\frac{\pi}{4}$ & & $\frac{\pi}{2}$ \\
\hline$z_{1}$ & 0.152 & 0.152 & $\frac{1}{4}$ & & $\frac{1}{8}$ \\
\hline $\operatorname{tr} L M^{+}$ & 2.77 & 2.77 & 2 & & 2 \\
\hline$m=3 k$ & $\{0,6 k-1\}$ & $\{0,6 k\}$ & $\{2 k-1,2 k\}$ & $\{2 k-$ & $1,4 k-1\}$ \\
\hline$x_{1}$ & $\frac{\pi}{6}$ & $\frac{\pi}{3}$ & $x_{1} \in\left[0, \frac{\pi}{6}\right]$ & & $\frac{\pi}{3}$ \\
\hline$x_{2}$ & - & - & $\frac{\arccos \left(\frac{-1}{2 \cos (2 x)}\right)}{2}$ & & - \\
\hline$z_{1}$ & $\frac{1}{6}$ & $\frac{1}{6}$ & $\frac{1}{4+8 \cos ^{2}(2 x)}$ & & $\frac{1}{4}$ \\
\hline $\operatorname{tr} L M^{+}$ & 2 & 2 & 4 & & $\frac{8}{3}$ \\
\hline$m=3 k$ & $\{2 k-1,4 k\}$ & $\{2 k-1,6 k-1\}$ & $\{2 k-1,6 k\}$ & $\{2 k$, & $4 k-1\}$ \\
\hline$x_{1}$ & 0.4531 & 0.6476 & $\frac{\pi}{3}$ & & $\frac{\pi}{6}$ \\
\hline$z_{1}$ & 0.12579 & 0.14 & $\frac{1}{5}$ & & $\frac{1}{4}$ \\
\hline $\operatorname{tr} L M^{+}$ & 3.4826 & 2.7044 & $\frac{25}{9}$ & & $\frac{8}{3}$ \\
\hline$m=3 k$ & $\{2 k, 4 k\}$ & $\{2 k, 6 k-1\}$ & $\{2 k, 6 k\}$ & $\{4 k$ & $-1,4 k\}$ \\
\hline$x_{1}$ & 1.1177 & $\frac{\pi}{6}$ & 0.9232 & & $\frac{\pi}{6}$ \\
\hline$z_{1}$ & 0.12579 & $\frac{1}{5}$ & 0.14 & & $\frac{1}{6}$ \\
\hline $\operatorname{tr} L M^{+}$ & 3.4826 & $\frac{25}{9}$ & 2.7044 & & 4 \\
\hline$m=3 k$ & $\{4 k-1,6 k-1\}$ & $\{4 k-1,6 k\}$ & $\{4 k, 6 k-1\}$ & $\{4 k, 6 k\}$ & $\{6 k, 6 k-1\}$ \\
\hline$x_{1}$ & $\frac{\pi}{5}$ & 0.94248 & 0.40397 & 1.16682 & $\frac{\pi}{4}$ \\
\hline$z_{1}$ & $\frac{3-\sqrt{5}}{4}$ & $\frac{3-\sqrt{5}}{4}$ & 0.14784 & 0.14784 & $\frac{1}{8}$ \\
\hline$z_{2}$ & - & - & - & - & $\frac{1}{8}$ \\
\hline $\operatorname{tr} L M^{+}$ & $\frac{3+\sqrt{5}}{2}$ & $\frac{3+\sqrt{5}}{2}$ & $\frac{3+\sqrt{5}}{2}$ & $\frac{3+\sqrt{5}}{2}$ & 4 \\
\hline
\end{tabular}

Table 1: The solutions of $x_{1}, x_{2}, z_{1}$ and $z_{2}$ of the the system (3.8). The L-optimal design for estimating the specific pair is specified in Theorem 4.1.

for all $t \in[-\pi, \pi]$, where $L^{(1)}=e_{1} e_{1}^{T}+e_{2} e_{2}^{T} \in R^{2 m+1 \times 2 m+1}$. We now apply Theorem 3.1 and obtain for the design $\xi_{3 k}^{s i n}$ and $L^{(k)}=e_{1} e_{1}^{T}+e_{2 k} e_{2 k}^{T} \in R^{2 m+1 \times 2 m+1}$

$$
\begin{aligned}
\operatorname{tr} L^{(k)} M^{+}\left(\xi_{3 k}^{s i n}\right) & =\operatorname{tr} L^{(1)} \bar{M}^{+}\left(\xi_{3}^{s i n}\right) \\
\varphi_{k}\left(t, \xi_{3 k}^{\text {sin }}\right) & =f^{T}(t) M^{+}\left(\xi_{3 k}^{\text {sin }}\right) L^{(k)} M^{+}\left(\xi_{3 k}^{\text {sin }}\right) f(t)=\varphi_{1}\left(k t, \xi_{3 k}^{\text {sin }}\right)
\end{aligned}
$$

where the function $\varphi_{1}\left(t, \xi_{3 k}^{s i n}\right)$ is defined in (3.10). Consequently $\varphi_{k}\left(t, \xi_{3 k}^{s i n}\right) \leq \operatorname{tr} L^{(k)} M^{+}\left(\xi_{3 k}^{s i n}\right)$ and it follows from Theorem 2.1 that the design $\xi_{3 k}^{s i n}$ is $L$-optimal for estimating the pair of the coefficients $\left\{\beta_{0}, \beta_{2 k-1}\right\}$ for any $k \geq 1$.

Our final result refers to $L$-optimal designs for estimating the coefficients in the Fourier regression model of degree $m=4 k$. It shows that the designs given in Theorem 3.1 are also 
$L$-optimal design for estimating other linear combinations of the coefficients in the model (1.1). The proof is obtained by similar arguments as given in the proof of Theorem 4.1 and therefore omitted.

Theorem 4.2 Consider trigonometric regression model (1.1) with $m=4 k$. The design $\xi^{*}=\xi_{4 k}^{c o s}$ is L-optimal for estimating the coefficients $\left\{\beta_{0}, \beta_{2 k-1}, \beta_{4 k}, \beta_{6 k-1} \beta_{8 k}\right\}$, where the parameters are given by $x_{1}=0.71686, z_{1}=0.112$ and $z_{2}=0.1084$ and are determined as the unique solution of the system (3.8)

Acknowledgements. This work has been supported in part by the Collaborative Research Center "Statistical modeling of nonlinear dynamic processes" (SFB 823) of the German Research Foundation (DFG). The work of the authors was also supported in part by an NIH grant award IR01GM072876:01A1 and by the BMBF project SKAVOE. The authors are also grateful to M. Stein, who typed parts of this paper with considerable technical expertise.

\section{References}

[1] Dette, H., Haller, G. (1998). Optimal Designs for the Identification of the Order of a Fourier Regression. Annals of Statistics, 26, 1496-1521.

[2] Dette, H. and Melas, V.B. (2003). Optimal designs for estimating individual coefficients in Fourier regression models. The Annals of Statistics, Vol. 31, 1669-1692.

[3] Dette, H., Melas, V.B. and Shpilev, P.V. (2007) Optimal designs for estimating the coefficients of the lower frequencies in trigonometric regression models. Annals of the Institute of Statistical Mathematics, 59(4), 655-673.

[4] Dette, H., Melas, V.B. and Shpilev, P.V. (2008) Optimal designs for estimating the pairs of coefficients in Fourier regression models. To appear in: Statistica Sinica.

[5] Hill, P.D.H. (1978). A note on the equivalence of D-optimal design measures for three rival linear models. Biometrika 65, 666-667.

[6] Karlin, S., Studden, W.J. (1966). Tchebycheff systems: with applications in analysis and statistics. Interscience, New York.

[7] Kiefer, J.C. (1974). General equivalence theory for optimum designs (approximate theory). The Annals of Statistics 2, 849-879.

[8] Lau, T.S., Studden, W.J. (1985). Optimal designs for trigonometric and polynomial regression. Ann. Statist. 13, 383-394. 
[9] Pázman, A. (1986). Foundations of Optimum Experimental Design. D. Reidel Publishing Company, Dordrecht.

[10] Pukelsheim, F., Rieder, S. (1992). Efficient rounding of approximate designs. Biometrika 79, 763-770.

[11] Pukelsheim, F. (1993). Optimal Design of Experiments. Wiley, New York.

[12] Rao, C.R. (1968). Linear statistical inference and its applications. Wiley, New York.

[13] Silvey, S.D. (1980). Optimal Design. Chapman and Hall, London.

[14] Wu, H. (2002). Optimal designs for first-order trigonometric regression on a partial circle. Statistica Sinica 12, 917-930.

[15] Zen, M.-M. and Tsai, M.-H. (2004). Criterion-robust optimal designs for model discrimination and parameter estimation in Fourier regression models. Journal of Statistical Planninng and Inference 124, 475-487. 

